\title{
The Hannover experience: Surgical treatment of tongue cancer - A clinical retrospective evaluation over a 30 years period
}

\author{
Horst Kokemueller ${ }^{\dagger}$, Majeed Rana ${ }^{*}$, Jennifer Rublack, Andre Eckardt, Frank Tavassol, Paul Schumann, \\ Daniel Lindhorst, Martin Ruecker and Nils-Claudius Gellrich
}

\begin{abstract}
Objectives: In this retrospective study, we present a clinical review of our experience with tongue cancer in order to obtain valid criteria for therapeutic decision-making.

Materials and methods: Between 1980 and 2009, a total of 341 patients with squamous cell carcinoma of the tongue were treated at our Department. The average follow-up was 5.2 years. 309 patients received surgical treatment, which was combined in nearly $10 \%$ with neoadjuvant and in nearly $20 \%$ with postoperative radio (chemo)therapy. 32 patients were excluded from surgery and received primary radiation.
\end{abstract}

Results: Local and regional failure occurred in $23.9 \%$ and $20.4 \%$, leading to a total failure rate of $37.2 \%$ after an average duration of 1,6 years. N-Status, extracapsular spread and clear margins were identified as the dominant factors for survival, which was calculated with $54.5 \%$ after 5 years.

Conclusions: We recommend categorical bilateral neck dissection in order to reliably remove occult lymph node metastases. Adjuvant treatment modalities should be applied more frequently in controlled clinical trials and should generally be implemented in cases with unclear margins and lymphatic spread.

Clinical relevance: This study provides new treatment strategies for primary tumour disease and for tumour recurrence.

Keywords: tongue cancer, squamous cell carcinoma, resection, survival, prognostic factors

\section{Introduction}

Tongue cancer is the most common malignancy diagnosed within the oral cavity, which accounts between 25 and $40 \%$ of oral squamous cell carcinomas [1]. Despite the development of multimodal treatment options, the prognosis remains relatively poor. Manifest and occult lymph node metastasis are observed more often than in any other cancer of the oral cavity [2]. The tongue seems to be predisposed for malignant invasion due to its highly muscularized structure and its rich lymphatic network [3]. Extensive resection with implementation of elective neck dissection especially in early stages of

\footnotetext{
* Correspondence: rana.majeed@mh-hannover.de † Contributed equally

Department for Oral and Maxillofacial Surgery, Hannover Medical School Carl-Neuberg-Str. 1, 30625 Hannover, Germany
}

tongue cancer has therefore been a source of debate in recent years [4-6].

Only a limited number of studies have examined larger series of tongue cancer. Spiro and Strong evaluated 314 patients (1957-1963) with tongue cancer and found an overall 5 -year survival rate of only $42 \%$ [7]. In a later study from the same institution with 412 patients (19691978), Callery et al. noted an increased proportion of female patients and an increased involvement of the base of tongue compared to the earlier decade [8]. More patients received primary and adjuvant radiotherapy, and elective neck dissection was performed more frequently. Age, sex, and adjuvant therapy did not affect survival, which remained stable compared to the earlier decade. However, lower stages of tongue cancer had a better prognosis when the tumour was located in the mobile tongue instead of the base of the tongue. In a

\section{() Biomed Central}


further study from the same institution with 297 patients (1978-1987), Franceschi et al. demonstrated an improved overall 5-year survival rate of $65 \%$, although the distribution of tumour stages was about the same compared to the preceding 10-year period [9]. Better survival was related to a more aggressive treatment of the neck even in early tumour stages and to adjuvant radiotherapy in advanced tumour stages. A considerable number of patients had to be upstaged after elective neck dissection due to occult lymph node metastases. The number of lymph node metastases turned out to be of prognostic value.

Since surgical treatment of tongue cancer strongly affects quality of life, many attempts have been made during the last decades towards organ preservation, leading to different treatment strategies with various combinations of surgery, radiation and chemotherapy [10]. Pernot et al. reviewed the medical records of 448 patients with tongue cancer who exclusively received radiation based therapy either as a combination of brachytherapy and external beam radiation or as a combination of brachytherapy and neck dissection [11]. The size of the lesion turned out to be the most important factor for prognosis with an overall 5-year survival rate of $44 \%$.

The purpose of the present study was to give a precise description of our experience with surgical based therapy of tongue cancer during the last three decades. Furthermore, prognostic factors for survival were analyzed in order to obtain valid criteria for therapeutic decision-making in clinical routine.

\section{Patients and methods}

Between January 1980 and December 2009, a total of 341 patients with squamous cell carcinoma of the tongue were treated at the Department of Oral and Maxillofacial Surgery, Hannover Medical School. Data concerning patient characteristics, clinical and pathologic tumour characteristics and treatment strategies and their results were obtained from a retrospective review of medical records. Informations regarding patient survival and local, regional and distant control were available for all patients. The average follow-up was 5.2 years. Statistical analysis for survival was calculated by the method of Kaplan and Meier. The relationship between the clinicopathologic variables and survival was assessed in univariate analysis using the log rank test. For multivariate analysis, the Cox proportional hazard model was used. A value of $\mathrm{p} \leq 0.05$ was considered to be statistically significant.

\section{Results}

The average age at diagnosis was 58.8 years, ranging between 19.2 and 96.5 years. There were 226 men and
115 women $($ male/female ratio $=2 / 1)$. The primary site was the tip of the tongue in 8 cases $(2.4 \%)$, dorsum of the tongue in 11 cases (3.2\%), the base of the tongue in 91 cases $(26.7 \%)$ and the lateral border of the mobile tongue in 231 cases (67.7\%). There was a strong correlation between the primary site and the tumour size, with increasing tumour size towards the base of the tongue. Tumour extension across the midline was observed in 33 cases (9.7\%). 14.5\% of tumours were graded as welldifferentiated, $69.6 \%$ as moderately-differentiated and $16.0 \%$ as poorly-differentiated. Anaplastic carcinomas were not observed. Nearly half of the patients suffered from T1-tumours (45.1\%), followed by T2-tumours (32.7\%) and T3- and T4-tumours (11.1\% each). 309 patients received surgical treatment, whereas 32 patients were excluded from surgical treatment and received primary radio(chemo)therapy after biopsy. These patients refused surgery, were in inappropriate condition for general anaesthesia or suffered from inoperable tumour disease. As a consequence, the proportion of advanced tumour stages was higher in this group. Detailed information of histopathological and clinical staging results (pT-status/cT-status) of patients with and without surgical treatment are given in Table 1. Clinical staging results were based on recorded clinical examinations and - if present - evaluation sheets of ultrasound (US) and computed tomography (CT). Data from modern imaging techniques of initial clinical staging was almost complete for the second half of the investigation period. In patients with surgical therapy, the neck was staged pN0, pN1, pN2 and pN3 in 48.5\%, 18.4\%, 14.9\% and $0.3 \%$ of cases. In 55 patients of this group (17.8\%) the neck was staged pNx due to missing surgical therapy of the neck. At the time of diagnosis, lymphadenectomy

Table 1 Histopathological and clinical staging results (pT-/cT-status) of patients with surgical treatment and patients with radiotherapy

\begin{tabular}{ccccc}
\hline group & T-stage & $\mathbf{n}$ & $\mathbf{\%}$ & valid\% \\
\hline \multirow{3}{*}{ surgical treatment } & pT1 & 150 & 48,5 & $\mathbf{4 9 , 0}$ \\
& pT2 & 108 & 35,0 & $\mathbf{3 5 , 3}$ \\
& pT3 & 31 & 10,0 & $\mathbf{1 0 , 1}$ \\
& pT4 & 17 & 5,5 & $\mathbf{5 , 6}$ \\
& total & 306 & 99,0 & $\mathbf{1 0 0 , 0}$ \\
& missing & 3 & 1,0 & \\
no9) & total overall & 309 & 100,0 & \\
& CT1 & 0 & 0,0 & $\mathbf{0 , 0}$ \\
& CT2 & 1 & 3,1 & $\mathbf{3 , 7}$ \\
& CT3 & 6 & 18,8 & $\mathbf{2 2 , 2}$ \\
& CT4 & 20 & 62,5 & $\mathbf{7 4 , 1}$ \\
& total & 27 & 84,4 & $\mathbf{1 0 0 , 0}$ \\
& missing & 5 & 15,6 & \\
& total overall & 32 & 100,0 & \\
\hline
\end{tabular}


was not considered necessary in these patients (cN0). In patients without surgical therapy, the presence of lymphatic spread was higher. In 10 patients of this group (31.3\%) the neck was staged $\mathrm{cNx}$ due to missing clinical data. Detailed information of histopathological and clinical staging results ( $\mathrm{pN}$-status/cN-status) of patients with and without surgical treatment are given in Table 2 . for both groups, there was a strong correlation between the tumour size at the primary site (T-status) and the presence of lymphatic spread (N-status). In the group of patients with surgical treatment, histologically assessed contralateral lymph node metastases were only observed in 9 patients $(2.9 \%)$ of whom 5 patients had 1,3 patients had 2 and 1 patient had 3 lymph node metastases on the contralateral side. Extracapsular spread was observed in $12.7 \%$ of patients with histologically assessed lymph node involvement, which strongly correlated with the degree of lymphatic spread ( $\mathrm{pN}$-status).

$9.7 \%$ of the operated patients received neoadjuvant radiochemotherapy (30Gy/Cisplatin) prior to surgery, which was performed via a transoral $(55.7 \%)$ or transmandibular approach $(32.6 \%)$ or in pull-through technique $(10.4 \%) .4$ patients $(1.3 \%)$ only received bilateral neck dissection, while the tumour at the primary site was radiated without surgery. Clear margins were achieved in $91.3 \%$ of the operated patients. On the ipsilateral neck, $44 \%$ of the operated patients received a comprehensive neck dissection, while $38.2 \%$ only received lymphadenectomy of level I-III and $17.8 \%$ no surgical therapy. On the contralateral neck, only $1.3 \%$ of these patients received a comprehensive neck dissection, while $36.6 \%$ still received lymphadenectomy of level I-III and the majority of $62.1 \%$ no surgical therapy. $19.5 \%$ of patients in the surgical group received postoperative radiation due to unclear margins, extensive tumour growth at the primary site, massive lymph node

Table 2 Histopathological and clinical staging results (pN-/cN-status) of patients with surgical treatment and patients with radiotherapy

\begin{tabular}{cccc}
\hline group & N-stage & $\mathbf{n}$ & $\%$ \\
\hline \multirow{3}{*}{ surgical treatment } & pN0 & 150 & 48,5 \\
$(\mathbf{n}=\mathbf{3 0 9})$ & $\mathrm{pN} 1$ & 57 & 18,4 \\
& $\mathrm{pN} 2$ & 46 & 14,9 \\
& $\mathrm{pN} 3$ & 1 & 0,3 \\
& $\mathrm{pNx}$ & 55 & 17,8 \\
non-surgical treatment & total & 309 & 100,0 \\
$(\mathbf{n}=\mathbf{3 2})$ & $\mathrm{cNO}$ & 0 & 0,0 \\
& $\mathrm{cN} 1$ & 8 & 25,0 \\
& $\mathrm{cN} 2$ & 13 & 40,6 \\
& $\mathrm{cN} 3$ & 1 & 3,1 \\
& $\mathrm{cNx}$ & 10 & 31,3 \\
& total & 32 & 100,0 \\
\hline
\end{tabular}

involvement or extracapsular spread, reflecting the scope of changing indications for radiotherapy during the past 30 years.

Local recurrence and regional recurrence were observed in 74 patients (23.9\%) and 63 patients (20.4\%) of the operated group, leading to total locoregional recurrence in 115 patients (37.2\%) after surgical based therapy. In patients with regional recurrence, secondary lymph node metastases were located on the ipsilateral neck in $73.8 \%$, on the contralateral neck in $18.0 \%$ and on both sides of the neck in $8.2 \%$. Locoregional recurrence occurred after an average duration of 1.6 years after initial treatment. $10.9 \%$ of all patients (surgical plus non-surgical group) developed a second malignant disease during follow-up.

The overall survival rates after $1,2,5$ and 10 years (including the surgical and non-surgical group) were calculated with $80.5 \%, 67.7 \%, 50.6 \%$ and $36.6 \%$. The survival rates of the surgical group were calculated with $83.8 \%, 71.5 \%, 54.5 \%$ and $39.6 \%$, whereas the survival rates of the non-surgical group were calculated with $47.8 \%, 30.7 \%, 13.7 \%$ and $6.8 \%$ (Figure 1, log rank $\mathrm{p}<$ $0.001)$. A detailed list of calculated survival rates for different $\mathrm{T}$ - and $\mathrm{N}$-stages are given in Table 3 and Table 4. In univariate analysis (log rank), the following factors were identified as prognostic factors for survival after surgical based therapy: tumour site (Figure 2, p = 0.005 ), grading (Figure 3, p $=0.004$ ), $\mathrm{pT}$-status (Figure $4, \mathrm{p}<0.001$ ), pN-status (Figure 5, p $<0.001$ ), number of lymph node metastases (Figure 6, p $<0.001$ ), extracapsular spread (Figure $7, \mathrm{p}<0.001$ ) and clear margins (Figure 8, p $<0.001$ ). Tumour extension across the

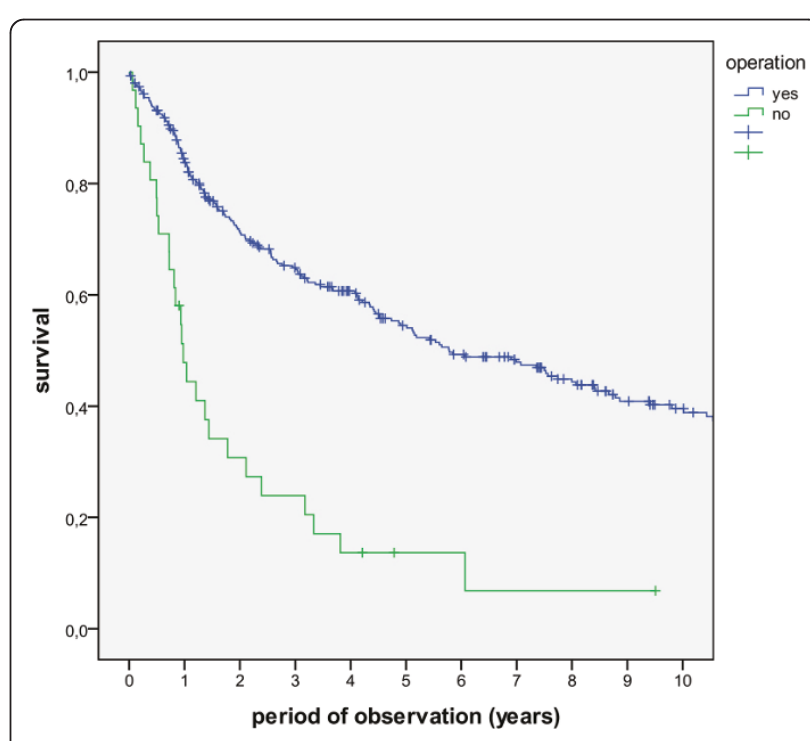

Figure 1 Survival of patients with surgical treatment and patients with primary radio(chemo)therapy (log rank $p<$ 0.001). 
Table 3 Survival rates of patients with different pT-stages (surgical group)

\begin{tabular}{|c|c|c|}
\hline pT-status & years & $\%$ \\
\hline & 1 & $91,9 \%$ \\
\hline \multirow[t]{4}{*}{ pT1 } & 2 & $85,3 \%$ \\
\hline & 5 & $70,2 \%$ \\
\hline & 10 & $56,5 \%$ \\
\hline & 1 & $80,9 \%$ \\
\hline \multirow[t]{4}{*}{$\mathrm{pT} 2$} & 2 & $62,1 \%$ \\
\hline & 5 & $42,7 \%$ \\
\hline & 10 & $25,2 \%$ \\
\hline & 1 & $63,0 \%$ \\
\hline \multirow[t]{4}{*}{ pT3 } & 2 & $48,5 \%$ \\
\hline & 5 & $21,0 \%$ \\
\hline & 10 & $16,8 \%$ \\
\hline & 1 & $64,7 \%$ \\
\hline \multirow[t]{3}{*}{ pT4 } & 2 & $52,9 \%$ \\
\hline & 5 & $44,1 \%$ \\
\hline & 10 & $26,5 \%$ \\
\hline
\end{tabular}

midline $(\mathrm{p}=0.356)$ and contralateral lymph node metastases $(\mathrm{p}=0.922)$ did not show significant values. For Nstatus, extracapsular spread and clear margins, significant values were also confirmed in multivariate analysis (Cox proportional hazard model).

Survival rates after 1, 2, 5 and 10 years for patients with and without local recurrence, regional recurrence and locoregional recurrence show significantly reduced

Table 4 Survival rates of patients with different pNstages (surgical group)

\begin{tabular}{ccc}
\hline pN-status & years & $\%$ \\
\hline \multirow{2}{*}{ pN0 } & 1 & $91,2 \%$ \\
& 2 & $83,2 \%$ \\
& 5 & $68,9 \%$ \\
pN1 & 10 & $49,6 \%$ \\
& 1 & $76,4 \%$ \\
& 2 & $60,7 \%$ \\
pN2 & 5 & $38,7 \%$ \\
& 10 & $24,8 \%$ \\
& 1 & $58,1 \%$ \\
pN3 & 2 & $26,6 \%$ \\
& 5 & $16,1 \%$ \\
& 10 & $8,1 \%$ \\
& 1 & $0,0 \%$ \\
& 2 & $0,0 \%$ \\
& 5 & $0,0 \%$ \\
& 10 & $0,0 \%$ \\
& 1 & $92,7 \%$ \\
& 2 & $87,1 \%$ \\
& 5 & $62,7 \%$ \\
& 10 & $48,7 \%$ \\
\hline
\end{tabular}

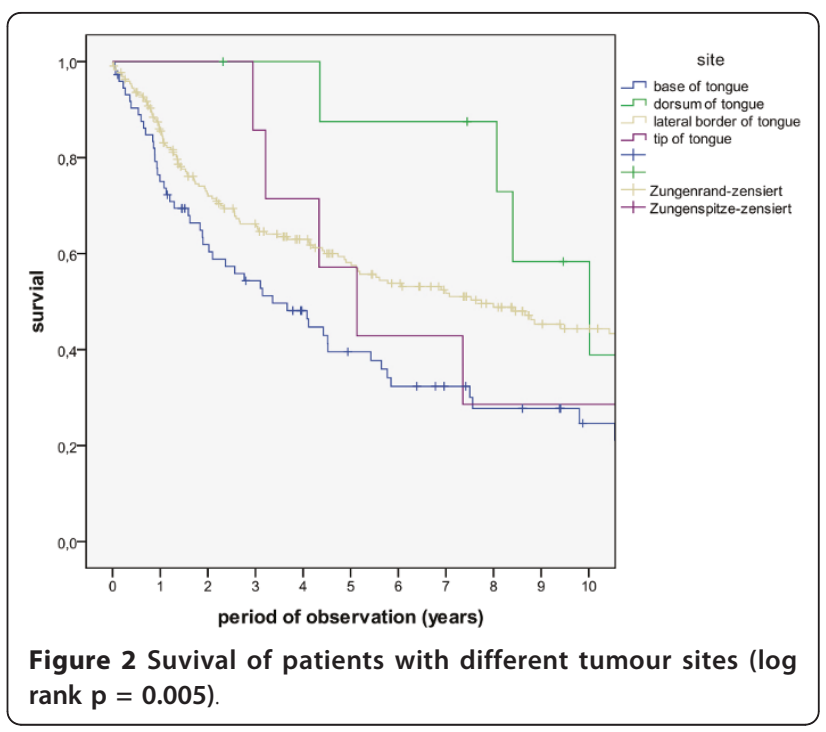

survival for patients with tumour recurrence (Figure 9, $\log$ rank $\mathrm{p}<0.001)$. Treatment modalities for tumour recurrence included singular surgery, surgery in combination with radiotherapy, singular radiation and combined radiotherapy, demonstrating a significant better prognosis when surgery was involved (Figure 10, log rank $\mathrm{p}<0.001)$.

\section{Discussion}

For many head and neck cancer patients, treatment consists of both chemotherapy and radiation therapy given simultaneously. This type of treatment is intensive and often results in serious and sometimes permanent damage to a patient's ability to swallow and hence, ability to eat a normal diet for the remainder of their lives.

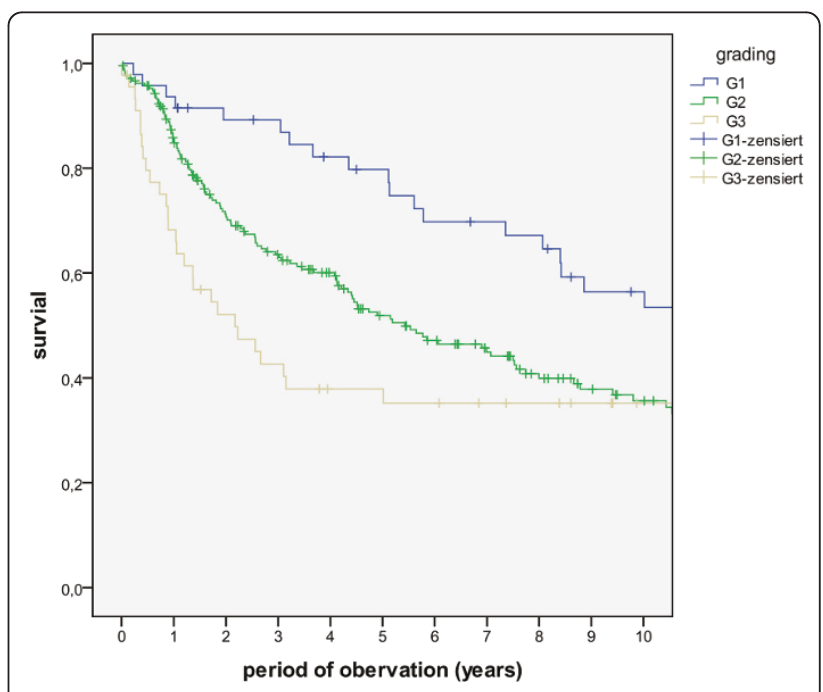

Figure 3 Survival of patients with different tumour grading (log rank $p=0.004)$. 


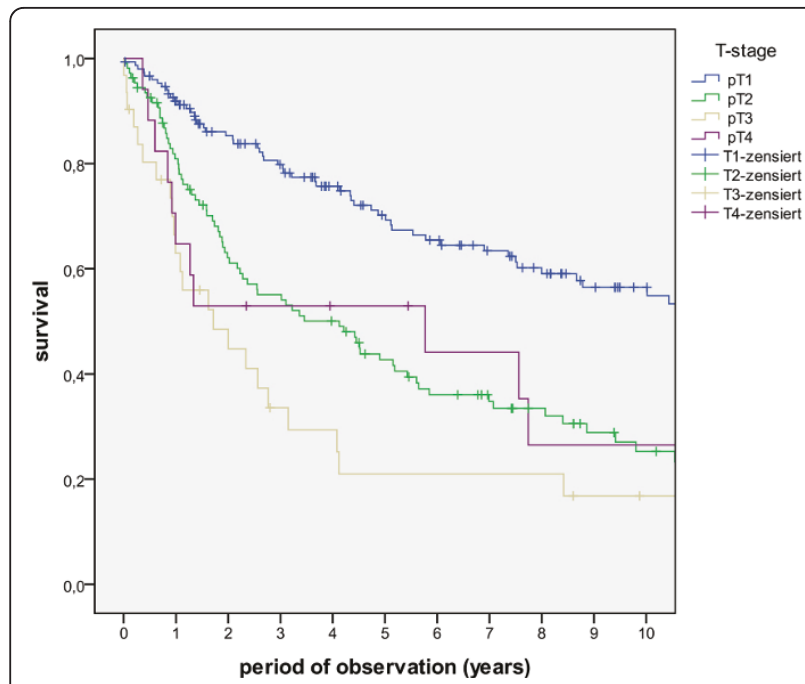

Figure 4 Survival of patients with different pT-Stage (log rank $p<0.001)$

The locoregional recurrence rate of our patients lies within the range of locoregional recurrence rates decribed by other authors, which are quoted between 16 and $42 \%$ [12-17]. According to the literature, adjuvant treatment modalities seem to improve locoregional control. Patients undergoing a targeted chemora- diation protocol for head and neck cancer lost about $10 \%$ of their pretreatment weight and had a decline in eating ability $[16,18]$. In our study, almost $10 \%$ of the operated patients received neoadjuvant radiochemotherapy prior to surgery and almost $20 \%$ of patients in the surgical group received postoperative radiation due to unclear margins, extensive tumour growth at the primary site, massive lymph node involvement or extracapsular

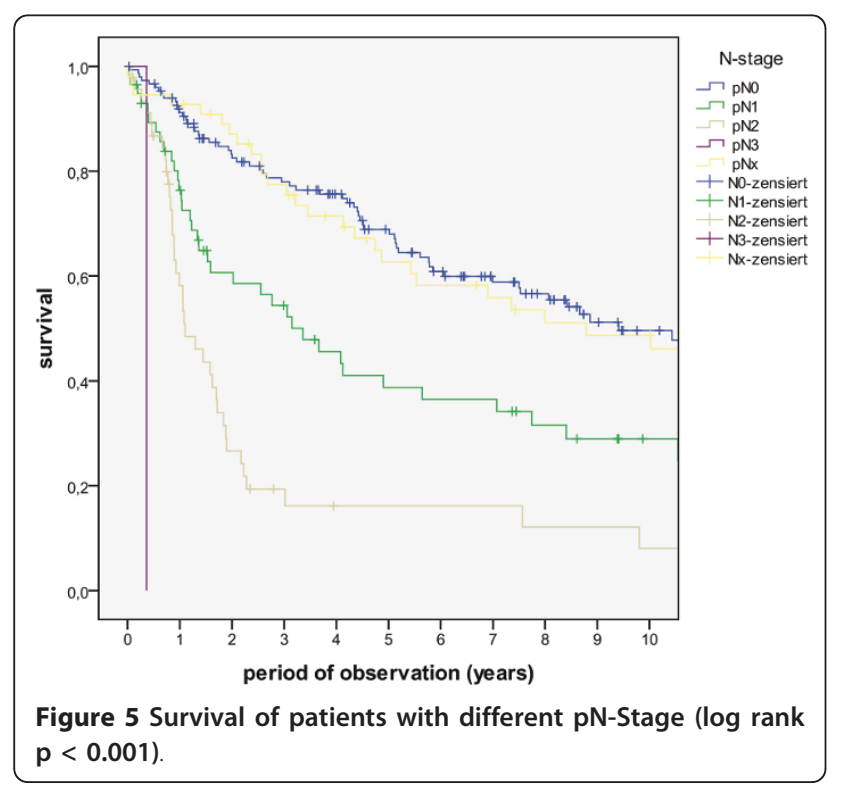

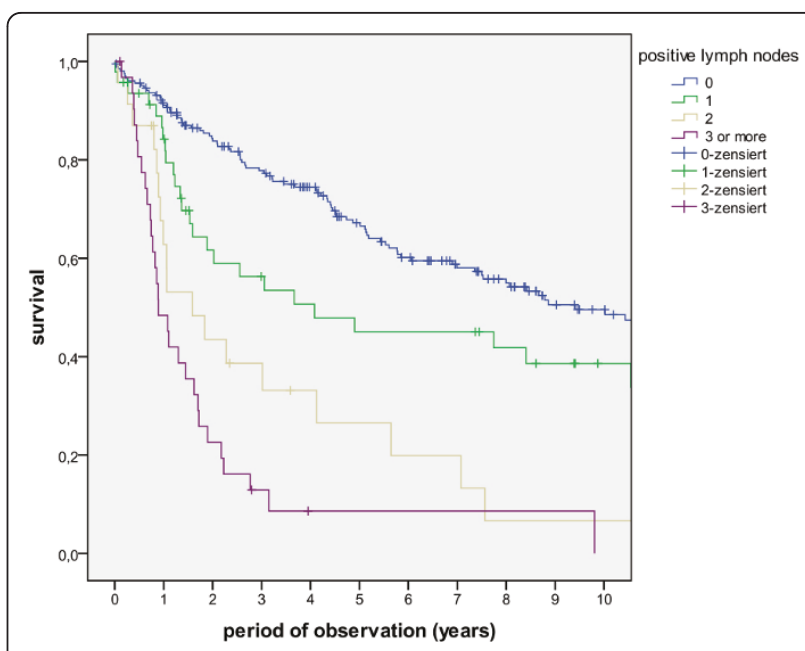

Figure 6 Survival of patients with different numbers of positive lymph nodes (log rank $p<0.001)$.

spread, reflecting the scope of changing indications for radiotherapy during a period of three decades. Since patient selection for (neo) adjuvant treatment was not randomized, the impact of radio(chemo)therapy could not be determined in our study.

Local failure at the primary site occurred in almost a quarter of our patients with surgical treatment, although clear margins were described for more than $90 \%$ of these patients. It is generally accepted that clear margins reduce local failure, although local control is not guaranteed. Byers et al. decribes local failure rates between 15 and $30 \%$ in patients with clear margins (> $5 \mathrm{~mm}$ distance to the tumour) and between 50 and $80 \%$ in patients with unclear margins [2]. In oncologic regard, it

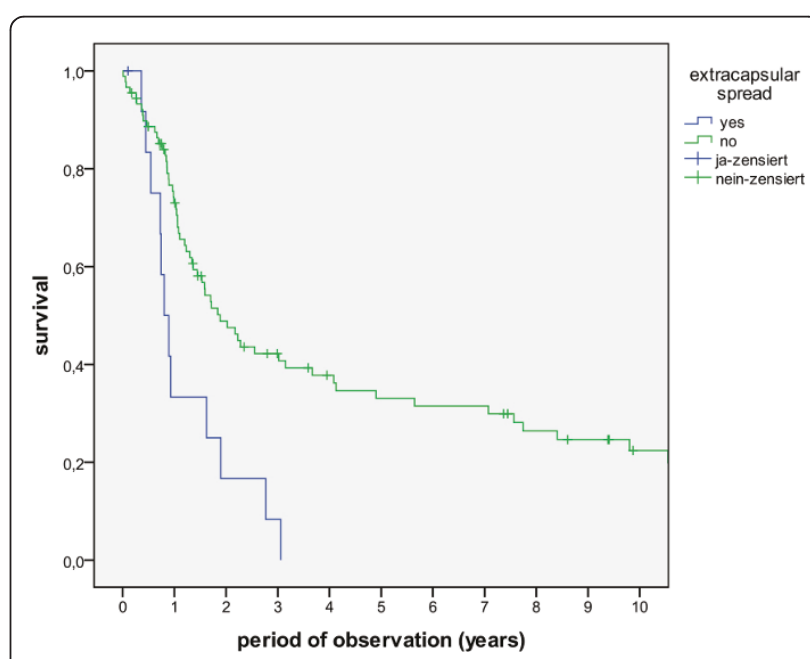

Figure 7 Survival of patients with and without extracapsular spread of positive lymph nodes (log rank $p<0.001$ ). 


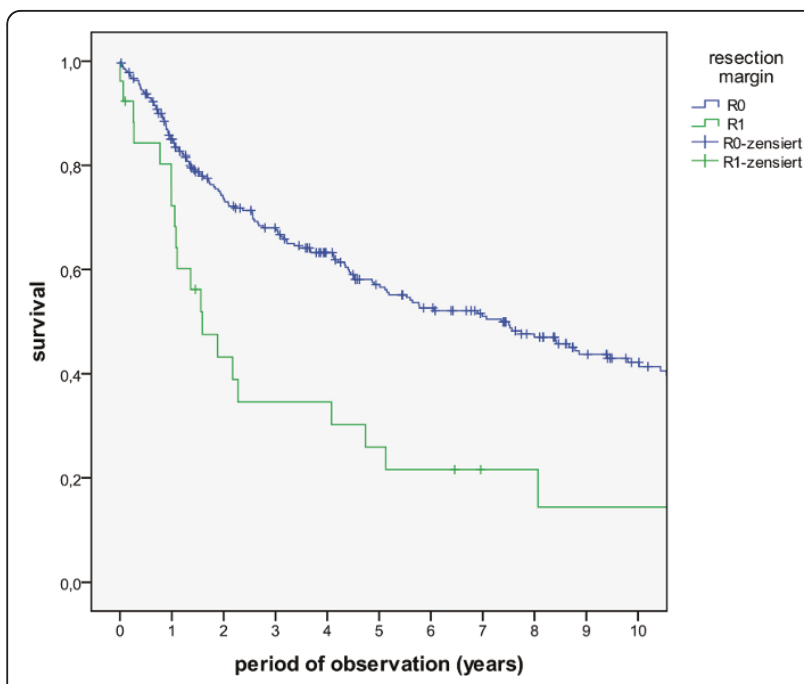

Figure 8 Survival of patients with and without clear margins (log rank $p<0.001)$.

also remains unclear how much distance to the tumour should be maintained in tongue cancer.

The dimension of lymphatic involvement seems to reflect the degree of malignancy in tongue cancer [19-23]. In multivariate analysis, $\mathrm{pN}$-status and extracapsular spread directly influenced survival besides clear margins. In univariate analysis, further factors showed prognostic value on the first sight. However, better survival of patients with tumours of the mobile tongue were attributed to the higher proportion of advanced tumour stages in patients with tumours of the base of the tongue, since these tumours were usually detected later $[24,25]$. The size of the tumour alone seemed not

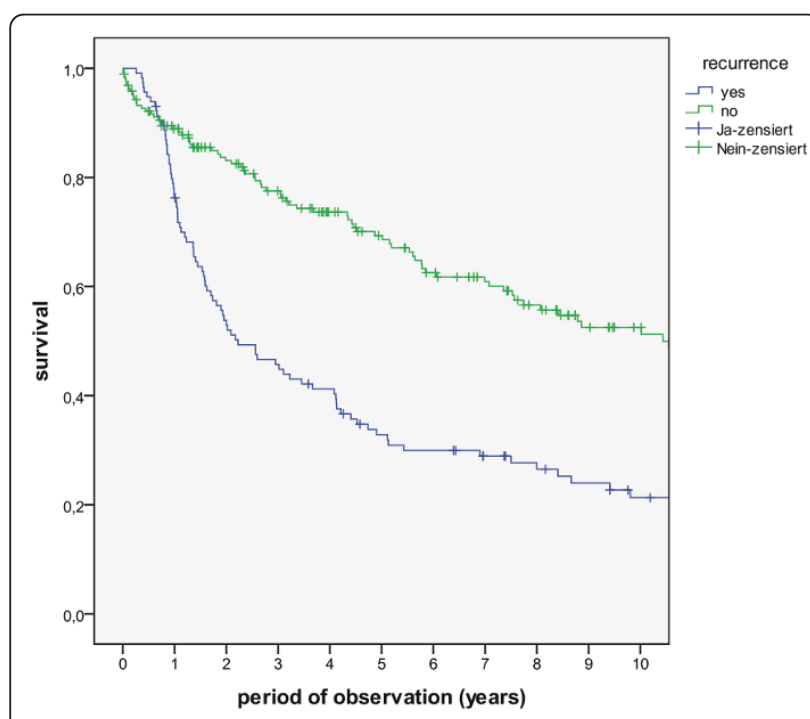

Figure 9 Suvival of patients with and without locoregional tumour recurrence $(\log$ rank $p<0.001)$.

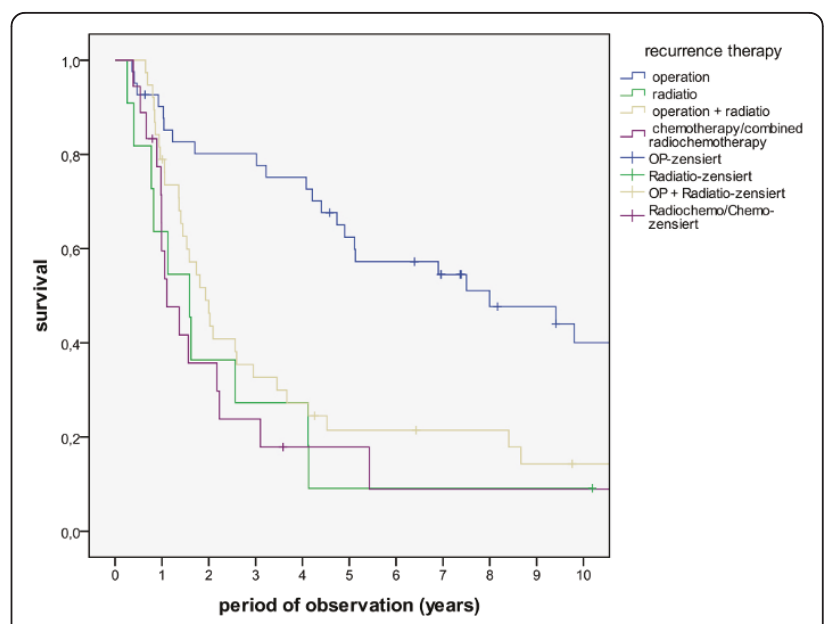

Figure 10 Survival of patients with different treatment modalities for locoregional tumour recurrence $(\log$ rank $p<$ 0.001).

to be of relevance for prognosis as long clear margins were obtained. This also explains why tumour extension across the midline alone did not affect survival [26,27]. Furthermore, the statistical distribution of tumour grade and number of lymph nodes were also associated with prognostic factors which were later identified in multivariate analysis. Age and gender - as described by other authors - did not influence prognosis in our study [24].

In the literature, the prognostic value of tumour grade is controversially discussed. Whereas some authors consider tumour grade as a prognostic factor [24,26-29], other authors doubt the prognostic value of tumour grade $[12,14,30-32]$. It seems reasonable that there are further prognostic factors, which are still unknown and currently not detectable by modern imaging and histopathological techniques [33]. Therefore, a clear definition of high risk groups remains incomplete up to the present. In current literature, serum and saliva are considered very useful in the fields of genomics, proteomics, transcriptomics and metabolomics for generation of diagnostic and prognostic biomarker signatures [34-36]. However, first studies for oral cancer show that these techniques seem to have greater potential as a tumor diagnostic tool for follow-up than for prognostication [37]. Further validation by multi-institutional studies and randomized clinical trials are recommended before these techniques can be translated into clinical practice for oral cancer [38].

It is generally accepted that oral cancer and especially cancer of the tongue often shows lymph node involvement even in early stages [Figure 11]. The proportion of occult metastases is quoted between 24 and $42 \%$ [39-41]. The number of patients with initial lymph node involvement in our study was low compared to other 


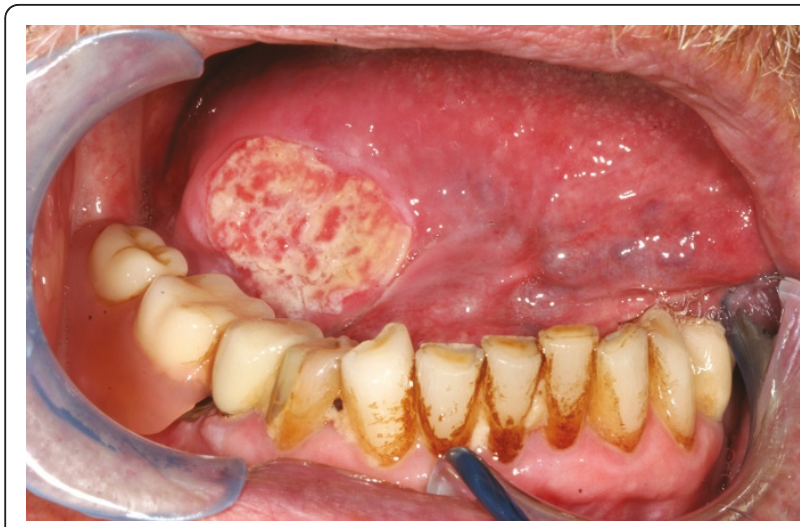

Figure 11 Toung carcinoma defect of the right toung in a 64 year-old patient following resection of tumor. The reconstruction is planed with an anterolateral thigh flap (ALTflap).

studies [42]. Especially the number of patients with bilateral lymphatic spread was surprisingly rare, since bilateral lymph node metastases are observed more frequently by other authors $[24,37,38]$. This might be attributed to the reduced proportion of patients in our study with unilateral and especially bilateral neck dissection during initial treatment. However, a regional failure rate in every fifth patient is a clear indicator for too restrictive surgical management of the neck. The reduced survival rates of patients with regional failure shows that a "wait and see" policy on the neck is clearly not advisable. Many authors therefore recommend elective neck dissection even in early stages of tongue cancer when the neck is clinically staged N0 $[17,39,40]$.

The overall survival rate of our patients with tongue cancer still lies within the range of survival rates decriped by other authors, which are quoted between 40 and $65 \%[7-9,11,24]$. We have to keep in mind that our study reviews a period of three decades and that treatment strategies have changed during this period towards a more aggressive course $[43,44]$. On the base of our results with high locoregional recurrence rates even in early stages of tongue cancer, we generally recommend extended resections on the primary site and categorical bilateral lymphadenectomy of at least level I-III in order to reliably remove occult lymph node metastases which can not be detected even by modern imaging techniques. In case of an open staging procedure with histologically approved lymph node metastases during surgery, a comprehensive neck dissection should complete lymphadenectomy [45]. As described before, neck dissection procedures are only associated with a low morbidity [46]. Modern reconstructive techniques with microvascular tissue transfer [Figure 12] help to keep functional impairment after partial glossectectomy tolerable and at least allow to refill substancial loss of soft tissue after

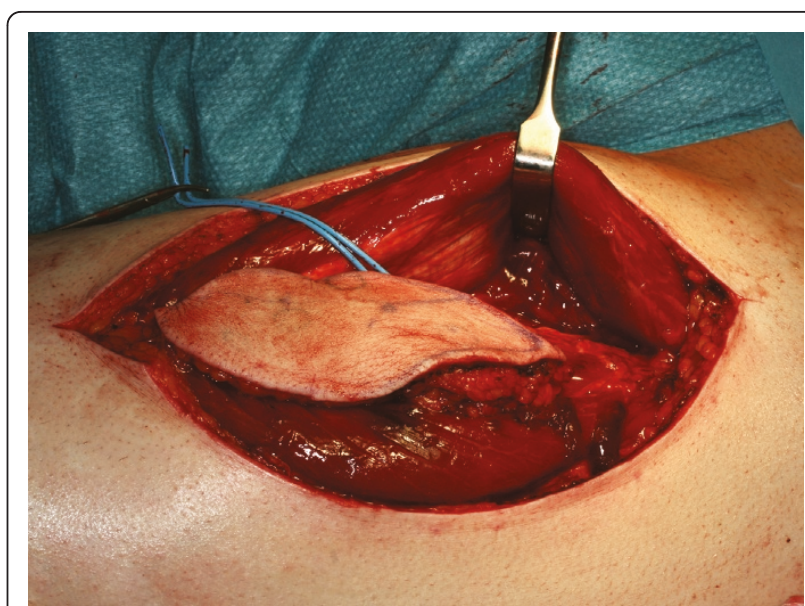

Figure 12 Harvested anterolateral thigh flap (ALT-flap) based on the perforator vessels of the descending branch of the lateral circumflex femoral artery for reconstruction of the right toung.

total glossectomy [47] [Figure 13]. According to our results, radical surgery also provides considerable survival rates for advanced stages of tongue cancer and should be recommended as treatment of first choice. Adjuvant treatment modalities should be applied more frequently in controlled clinical trials and should generally be implemented in cases with unclear margins and lymphatic spread.

In general, treatment strategies for tumour recurrence follow the same principles than for primary tumour disease. As already described by Eckardt et al., surgical intervention seems to be associated with better survival for patients with recurrent floor of mouth carcinoma, which was also confirmed by our study for patients with tongue cancer [48]. Therefore, curative total resection should be aimed if survival is clearly defined as highest

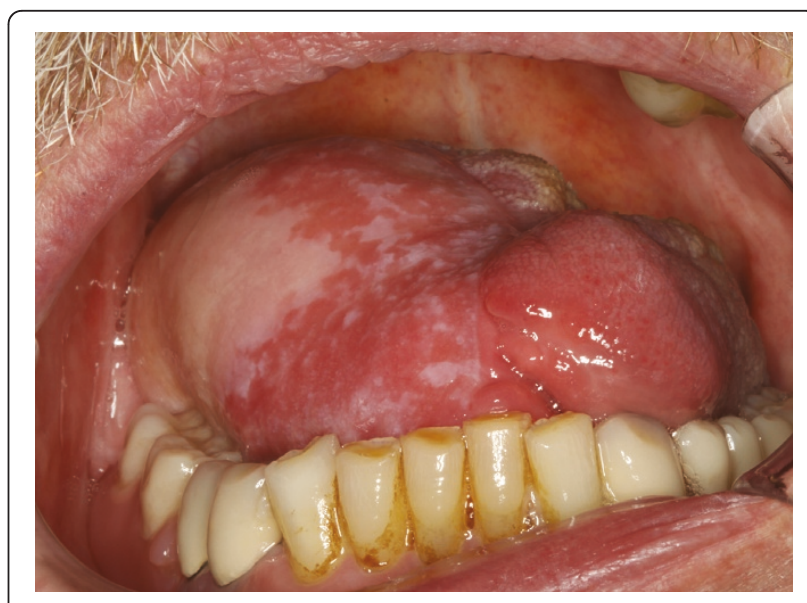

Figure 13 Reconstructed defect of the toung. Final result of the anterolateral thigh flap (ALT flap) after 6 month. 
preference. However, functional impairment with dramatic loss of life quality needs to be discussed individually with every patient. If recurrent tumour disease seems to be unresectable, subtotal tumour reduction can be discussed in order to improve the starting position for adjuvant treatment modalities in an interdisciplinary treatment concept. In general, however, mutilations caused by surgical interventions should be minimized in these cases in order to preserve the greatest amount of life quality as long as possible.

\section{Acknowledgements}

Funding: The article processing charges are funded by the Deutsche Forschungsgemeinschaft (DFG), "Open Acess Publizieren".

\section{Authors' contributions}

HK and MR contributed equally to this work. JR, AE, FT, PS, DL, MRu and NCG conceived of the study and participated in its design and coordination. HK and MR drafted the manuscript. All authors read and approved the final manuscript.

\section{Competing interests}

The authors declare that they have no competing interests.

Received: 11 April 2011 Accepted: 21 May 2011 Published: 21 May 2011

\section{References}

1. Regezi JA, Sciubba JJ, Jordan RCK: Oral pathology: clinical, pathologic correlations.Edited by: St Louis (MO). Saunders Elsevier; , 5 2008:12-24

2. Byers RM, El-Naggar AK, Lee YY, et al: Can we detect or predict the presence of occult nodal metastases in patients with squamous carcinoma of the oral tongue? Head Neck 1998, 20:138-44

3. Lim MSRE: Correlational of oral tongue cancer inversion with matrix metalloproteinases (MMPs) and vascular endothelial growth factor (VEGF) expression, by. In J Surg Oncol Edited by: Kim S-H, Cho NH, Kim K 2006, 93:253-54

4. Huang SF, Kang CJ, Lin CY, et al: Neck treatment of patients with early stage oral tongue cancer: comparison between observation, supraomohyoid dissection, and extended dissection. Cancer 2008, 112:1066-75

5. D'Cruz AK, Siddachari RC, Walvekar RR, et al: Elective neck dissection for the management of the NO neck in early cancer of the oral tongue: need for a randomized controlled trial. Head Neck 2009, 31:618-24.

6. Hsu MY, Wang CC: Elective radiotherapy or neck dissection for CT-staged T1-2N0 oral tongue cancer. Head Neck 2010, 32(2):191-8.

7. Spiro RH, Strong EW: Surgical treatment of cancer of the tongue. Surg Clin North Am 1974, 54:759-65.

8. Callery CD, Spiro RH, Strong EW: Changing trends in the management of squamous carcinoma of the tongue. Am J Surg 1984, 148:449-54.

9. Franceschi D, Gupta R, Spiro RH, Shah JP: Improved survival in the treatment of squamous carcinoma of the oral tongue. Am J Surg 1993, 166:360-65.

10. Khalilur RM, Hayashi K, Shibuya H: Brachytherapy for tongue cancer in the very elderly is an alternative to external beam radiation. Br J Radiol 2010.

11. Pernot M, Malissard L, Hoffstetter S, et al: The study of tumoral, radiobiological and general health factors that influence results and complications in a series of 448 oral tongue carcinomas treated exclusively by irradiation. Int J Radiat Oncol Biol Phys 1994, 29:673-79.

12. El-Husseiny G, Kandil A, Jamshed A, et al: Squamous cell carcinoma of the oral tongue: An analysis of prognostic factors. $\mathrm{Br} J$ Oral Maxillofac Surg 2000, 38:193-99.

13. Hosal AS, Unal OF, Ayhan A: Possible prognostic value of histopathologic parameters in patients with carcinoma of the oral tongue. Eur Arch Otorhinolaryngol 1998, 225:216-19.
14. Kantola S, Parikka M, Jokinen $\mathrm{K}$, et al: Prognostic factors in tongue cancer: Relative importance of demographic clinical and histopathological factors. Br J Cancer 2000, 83:614-19.

15. Ünal OF, Ayhan A, Hosal AS: Prognostic value of p53 expression and histopathological parameters in squamous cell carcinoma of oral tongue. J Laryngol Otol 1999, 113:446-50.

16. Wang YH, Chen YF, Guo ZM, Zhang Q, Liu TR, Chen FJ: Reasons for recurrence and prognostic analysis of early stage squamous cell carcinoma of the oral tongue. Chinese Journal of Cancer 2009, 28:1-5.

17. Zwetyenga N, Majoufre-Lefebvre C, Siberchicot F, Demeaux H, Pinsolle J: Squamous cell carcinoma of the tongue: treatment results and prognosis. Rev Stomatol Chir Maxillofac 2003, 104:10-17.

18. Hua $H$, Zeng $Z Y, X u G P$ : Multivariate analysis of prognosis of patients with clinical stage I and II carcinoma of mobile tongue. Ai Zheng 2003, 22:210-13

19. Daisuke S, Myers JN: Metastasis of squamous cell carcinoma of the oral tongue. Cancer Metastasis Rev 2007, 26:645-62.

20. Greenberg JS, Fowler R, Gomez J, et al: Extent of extracapsular spread: A critical prognosticator in oral tongue cancer. Cancer 2003, 97:1464-70.

21. Okamoto M, Nishimine M, Kishi M, et al: Prediction of delayed neck metastasis in patients with stage $\mathrm{I} / \mathrm{Il}$ squamous cell carcinoma of the tongue. J Oral Pathol Med 2002, 31:227-33.

22. Myers JN, Greenberg JS, Mo V, Roberts D: Extracapsular spread. A significant predictor of treatment failure in patients with squamous cell carcinoma of the tongue. Cancer 2001, 92:3030-36.

23. Shingaki S, Takada M, Sasai K, et al: Impact of lymph node metastasis on the pattern of failure and survival in oral carcinoma. Am J Surg 2003, 185:278-84.

24. Gorsky M, Epstein JB, Oakley C, Le ND, Hay J, Stevenson-Moore P: Carcinoma of the tongue: A case series analysis of clinical presentation, risk factors, staging, and outcome. Oral Surg Oral Med Oral Pathol Oral Radiol Endod 2004, 98:546-52

25. Malone JP, Stephens JA, Grecula JC, Rhoades CA, Ghaheri BA, Schuller DE: Disease control, survival, and functional outcome after multimodal treatment for advanced-stage tongue base cancer. Head Neck 2004 26:561-72

26. Korpi JT, Kervinen V, Maklin H, et al: Collagenase-2 (matrix metalloproteinase-8) plays a protective role in tongue cancer. $\mathrm{Br} J$ Cancer 2008, 98:766-75

27. Teixeira G, Antonangelo L, Kowalski L, Saldiva P, Ferraz A, Silva Filho G: Argyrophilic nucleolar organizer regions staining is useful in predicting recurrence-free interval in oral tongue and floor of mouth squamous cell carcinoma. Am J Surg 1996, 172:684-88,

28. Arduino PG, Carrozzo M, Chiecchio A, et al: Clinical and histopathologic independent prognostic factors in oral squamous cell carcinoma: a retrospective study of 334 cases. J Oral Maxillofac Surg 2008, 66:1570-79.

29. Fan $\mathrm{KH}$, Lin CY, Kang CJ: Combined-modality treatment for advanced oral tongue squamous cell carci-noma. Int I Radiat Oncol Biol Phys 2007, 67:453-61

30. Kademani D, Bell RB, Bagheri S, et al: Prognostic factors in intraoral squamous cell carcinoma: the influence of histologic grade. J Oral Maxillofac Surg 2005, 63:1599-605.

31. Al-Rajhi N, Khafaga Y, El-Husseiny J, et al: Early stage carcinoma of oral tongue: prognostic factors for local control and survival. Oral Oncol 2000, 36:508-14

32. O-charoenrat P, Pillai $G$, Patel $S$, et al: Tumour thickness predicts cervical nodal metastases and survival in early oral tongue cancer. Oral Oncol 2003, 39:386-90

33. Woolgar JA: Histopathological prognosticators in oral and oropharyngeal squamous cell carcinoma. Oral Oncol 2006, 42:229-39.

34. Nagler RM: Saliva as a tool for oral cancer diagnosis and prognosis. Oral Oncol 2009, 45:1006-10

35. Matharoo-Ball B, Miles AK, Creaser CS, Ball G, Rees R: Serum biomarker profiling in cancer studies: a question of standardisation? Vet Comp Oncol 2008, 6:224-47.

36. Zhang L, Farrell JJ, Zhou H, et al: Salivary transcriptomic biomarkers for detection of resectable pancreatic cancer. Gastroenterology 2010, 138:949-57.

37. Bello IO, Soini Y, Salo T: Prognostic evaluation of oral tongue cancer: Means, markers and perspectives (I). Oral Oncology 2010, 46:630-635. 
38. Bello IO, Soini Y, Salo T: Prognostic evaluation of oral tongue cancer: Means, markers and perspectives (II). Oral Oncology 2010, 46:636-643.

39. Brugere JM, Mosseri VF, Mamelle $G$, et al: Nodal failures in patients with $\mathrm{No}, \mathrm{N}+$ oral squamous cell carcinoma without capsular rupture. Head Neck 1996, 18:133-37.

40. Kowalski LP, Sanabria A: Elective neck dissection in oral carcinoma: a critical review of the evidence. Acta Otorhinolanyngol Ital 2007, 27:113-17.

41. Manni JJ, van der Hoogen FJ: Supraomohyoid neck dissection with frozen section biopsy as a staging pro-cedure in the clinically node-negative neck in carcinoma of the oral cavity. Am J Surg 1991, 162:373-76.

42. Capote-Moreno A, Naval L, Munoz-Guerra MF, Sastre J, RodríguezCampo FJ: Prognostic factors influencing contralateral neck lymph node metastases in oral and oropharyngeal carcinoma. J Oral Maxillofac Surg 2010, 68:268-75.

43. Ho CM, Lam KH, Wei WI, Lau SK, Lam LK: Occult lymph node metastasis in small oral tongue cancers. Head Neck 1992, 14:359-63.

44. Lydiatt DD, Robbins KT, Byers RM, Wolf PF: Treatment of stage I and II oral tongue cancer. Head Neck 1993, 15:308-12.

45. Persky MS, Lagmay VM: Treatment of the clinically negative neck in oral squamous cell carcinoma. Laryngoscope 1999, 109:1160-64.

46. Kokemüller $\mathrm{H}$, Brachvogel P, Eckardt A, Hausamen JE: Neck dissection in oral cancer - clinical review and analysis of prognostic factors. Int I Oral Maxillofac Surg 2002, 31:608-614.

47. Yun IS, Lee DW, Lee WJ, Lew DH, Choi EC, Rah DK: Correlation of neotongue volume changes with functional outcomes after long-term follow-up of total glossectomy. J Craniofac Surg 2010, 21:111-16.

48. Eckardt A, Barth EL, Kokemueller H, Wegener G: Recurrent carcinoma of the head and neck: treatment strategies and survival analysis in a 20year period. Oral Oncology 2004, 40:427-432

doi:10.1186/1758-3284-3-27

Cite this article as: Kokemueller et al: The Hannover experience:

Surgical treatment of tongue cancer - A clinical retrospective evaluation over a 30 years period. Head \& Neck Oncology 2011 3:27.

\section{Submit your next manuscript to BioMed Central and take full advantage of:}

- Convenient online submission

- Thorough peer review

- No space constraints or color figure charges

- Immediate publication on acceptance

- Inclusion in PubMed, CAS, Scopus and Google Scholar

- Research which is freely available for redistribution

Submit your manuscript at www.biomedcentral.com/submit 